\title{
Proliferative retinopathy and maculopathy are two independent conditions in sickle cell disease: Is there a role of blood rheology?
}

Laurence Beral $^{\mathrm{a}, \mathrm{b}}$, Nathalie Lemonne ${ }^{\mathrm{c}}$, Marc Romana $^{\mathrm{b}, \mathrm{d}}$, Keyne Charlot $^{\mathrm{b}, \mathrm{d}, \mathrm{e}}$, Marie Billaud $^{\mathrm{c}}$, Malik Acomat ${ }^{\mathrm{a}}$, Coralie Zorobabel ${ }^{\mathrm{a}}$, Elie Nader ${ }^{\mathrm{f}}$, Maryse Etienne-Julan $^{\mathrm{c}}$, Thierry David ${ }^{\mathrm{a}}$ and Philippe Connes $\mathrm{s}^{\mathrm{b}, \mathrm{d}, \mathrm{f}, \mathrm{g}, *}$

${ }^{a}$ Service d'ophtalmologie, CHU de Pointe-à-Pitre/Abymes, route de Chauvel, Pointe-à-Pitre, Guadeloupe, France

${ }^{\mathrm{b}}$ Université des Antilles, CHU de Pointe-à-Pitre, Guadeloupe, Université Sorbonne Paris Cité, Université Paris Diderot, Inserm, INTS, Unité Biologie Intégrée du Globule Rouge UMR_S1134, Paris, France

${ }^{\mathrm{c}}$ Unité transversale de la Drépanocytose, $\mathrm{CHU}$ de Pointe-à-Pitre/Abymes, route de Chauvel, Pointe-à-Pitre, Guadeloupe, France

${ }^{\mathrm{d}}$ Laboratoire d'Excellence GR-Ex «The Red Cell: From Genesis to Death», PRES Sorbonne Paris Cité, Paris, France

${ }^{\mathrm{e}}$ Unité Physiologie des Exercices et Activités en Conditions Extrêmes, Institut de Recherche Biomédicale des Armées, Brétigny-sur-Orge, France

${ }^{\mathrm{f}}$ Laboratoire Interuniversitaire de Biologie de la Motricité (LIBM), EA7424, Equipe Biologie Vasculaire et du Globule Rouge, Université Claude Bernard Lyon 1, COMUE Lyon, Villeurbanne, France

${ }^{\mathrm{g}}$ Institut Universitaire de France, Paris, France

\begin{abstract}
.
OBJECTIVE: Our study investigated the prevalence of retinopathy and maculopathy in sickle cell patients and tested the association between these two conditions. In addition, we tested whether hematological and hemorheological parameters, as well as genotype, were involved in the development of these two conditions.

METHODS: Seventy sickle cell adult patients were recruited: 37 with sickle cell anemia (SCA) and 33 with sickle cell hemoglobin $\mathrm{C}$ disease (SCC). All patients underwent retinal examination and macular ocular coherence tomography. Blood was sampled for the measurements of hematological and hemorheological parameters.

RESULTS: Twenty-six patients had maculopathy and 30 had retinopathy with no significant difference between SCA and SCC patients. No association between the presence of retinopathy and maculopathy was detected. RBC aggregation was higher and RBC deformability lower at $3 \mathrm{~Pa}$ in SCA patients. Blood viscosity and hematocrit were higher in SCC than in SCA patients. However, no association was found between biological parameters and the sickle ocular complications studied. CONCLUSIONS: Our study showed that retinopathy and maculopathy are common in sickle cell disease. Nevertheless, we found no association with hematological parameters, blood rheology or genotype.
\end{abstract}

Keywords: Hematology, hemorheology, maculopathy, retinopathy, sickle cell disease

\footnotetext{
${ }^{1}$ No author has proprietary interest.

*Corresponding author: Philippe Connes, E-mails: pconnes@yahoo.fr and philippe.connes@univ-lyon1.fr.
} 


\section{Introduction}

Retinopathy is a common complication of sickle cell disease (SCD) [1]. The National Institute of Health (NIH) recommends that all patients with SCD should have regular dilated fundoscopy by an ophthalmologist with expertise in retinal diseases [2]. As a matter of fact, fundus examination may reveal microcirculatory alterations. Retinopathy is more frequently encountered in patients with sickle cell-hemoglobin $\mathrm{C}$ disease (SCC) than in patients with sickle cell anemia (SCA) [3]. The greater whole blood viscosity usually reported in SCC patients in comparison with SCA patients has been hypothesized to increase the risk of SCC patients to develop retinopathy $[4,5]$. In addition, Serjeant et al. reported higher whole blood viscosity in SCA males with retinopathy than in SCA males without retinopathy [6]. It has also been reported that high hemoglobin concentration and low fetal hemoglobin level could increase the risk of SCA patients to develop retinopathy [7-9]. In SCC females, high mean cell volume and low fetal hemoglobin have been reported to be associated with retinopathy [7, 10].

In contrast, maculopathy has been poorly investigated in SCD but recent studies indicate that this complication could be frequent in this disease [11-13]. Han et al. reported $43 \%$ of macular thinning among their SCD population and Mathew et al. found 44\% of 107 SCD patients having maculopathy $[12,14]$. In most of these studies, maculopathy in SCD was detected using a cross-sectional spectral domain tomography (SD-OCT) as a thinning of the temporal macula, with selective loss of the retinal ganglion cell and nerve fiber layer. The thinning may reflect chronic ischemia of the retinal ganglion cells and nerve fibers coursing temporally as they head towards the optic nerve [13]. However, the mechanisms at the origin of maculopathy in SCD have not been investigated until now. Because maculopathy is suggested to result from ischemic events [13] and because blood rheology is severely impaired in SCD [15], the aim of the present study was to compare hematological and hemorheological parameters between SCD (SCA and SCC) patients with maculopathy and those without [16]. In addition, we analyzed the association between maculopathy and retinopathy in SCA and SCC patients.

\section{Methods}

Seventy adult patients with SCD (37 SCA and 33 SCC) regularly followed by the sickle cell unit of the academic hospital of Pointe-à-Pitre (Guadeloupe, French West Indies) were included between January 2014 and December 2016. All patients were at steady state at the time of the study (i.e. without vaso-occlusive crisis, acute medical complication or blood transfusion/phlebotomies within the last 3 months). Any condition causing a peripheral proliferative retinopathy (i.e., diabetes, central retinal vein or artery occlusion) was exclusion criteria. Twenty-two SCA $(59,4 \%)$ patients were treated by hydroxyurea while none of the SCC patients received this therapy. The information related to their medical history (vaso-occlusive crisis, acute chest syndrome, hypertension, leg ulcers, nephropathy, osteonecrosis) were collected over the year preceding the inclusion. Blood was sampled in EDTA tubes for hematological and hemorheological measurements. This study received the ethical approval of the Regional Ethics Committee (CPP Sud-Ouest Outre-Mer III, Bordeaux, France). The experiments were performed in accordance with the guidelines set by the Declaration of Helsinki. Informed consent was obtained from the subjects after explanation of the nature of the study.

\subsection{Ophthalmic measurements}

Two independent ophthalmologists performed indirect ophthalmoscopy with a non-contact slip lamp lens (Super-Field, Volk Optical, Mentor, OH, USA). SD-OCT data were acquired using a Copernicus SD-OCT (Copernicus, Optopol Technologies, Zawierci, Poland). No patient needed fluorescein 
angiography for further investigation. The patients were classified according to their genotype (SCA or SCC) and according to the presence/absence of retinopathy or maculopathy.

\subsection{Hematological and hemorheological measurements}

Hemorheological measurements were performed immediately after blood sampling to avoid any red blood cell alterations and were conducted in agreement with the most recent International recommendations for blood rheological analyses [16]. We measured whole blood viscosity with a cone-plate viscometer (Brookfield DVII + with CPE40 spindle) at $25^{\circ} \mathrm{C}$ and two shear rates: 90 and $225 \mathrm{~s}^{-1}$. The ability of red blood cell (RBC) to deform under flow (i.e., RBC deformability) was studied by laser diffraction analysis (Ektacytometry, LORCA, RR Mechatronics, Hoorn, The Netherlands) at $37^{\circ} \mathrm{C}$ and at two shear stresses: $3 \mathrm{~Pa}$ and $30 \mathrm{~Pa}$. The system calculates an average $\mathrm{RBC}$ elongation index (EI). The higher this index, the more deformable the red blood cells (RBC). After adjustment of hematocrit to $40 \%$, the LORCA (RR Mechtronics, Hoom, The Netherlands) was used to determine RBC aggregation properties at $37^{\circ} \mathrm{C}$. The system uses syllectometry (i.e, laser backscatter versus time) to calculate an index of RBC aggregation index (AI). The RBC disaggregation threshold - i.e. the minimal shear rate needed to breakdown RBC aggregates - was determined using a re-iteration procedure. Hematocrit (Hct) was determined by blood microcentrifugation (Jouan-Hema-C, Saint Herblain, France). Total counts of white blood cells (WBC), platelets and RBCs, mean corpuscular volume (MCV), hemoglobin concentration $(\mathrm{Hb})$ were determined using a hematology analyzer (Max M-Retic, Coulter, USA). An automatic analyser (Hitachi 912, Roche Ltd.) photometrically measured Lactate dehydrogenase (LDH). A solid phase sandwich immunoassay (Phase Range canine C-Reactive Protein Assay, Tridelta Ltd.) was used for quantitative analysis of CRP. Polymerase Chain Reaction (GAP-PCR) was used to detect six common $\alpha$-thalassemia deletions $[17,18]$.

\subsection{Statistical analysis}

Hematological and hemorheological parameters were studied in the whole SCD group (SCA + SCC patients) and separately in SCA and SCC patients. We tested the associations between the occurrence of retinopathy and maculopathy as well as with $\alpha$-thalassemia, hydroxyurea therapy and the different complications collected in the medical files, using a $\mathrm{Chi}^{2}$ test. Hematological and hemorheological parameters were compared between patients with or without retinopathy or maculopathy in SCA and SCC groups using an unpaired student $t$ test. Significance level was set at $p<0.05$. Analyses were conducted using GraphPad Prism (version $6.0 \mathrm{c}$ ).

\section{Results}

Of the 70 SCD patients included, $26(37.1 \%)$ had maculopathy and $30(42.9 \%)$ had retinopathy with no significant difference between SCA and SCC patients (Table 1). Nine SCA (26.5\%) and 2 SCC (6.9\%) patients had both maculopathy and retinopathy. In the whole cohort, 15 patients showed maculopathy without retinopathy and 17 had retinopathy without maculopathy. In SCA patients, 9 had retinopathy alone and 7 had maculopathy alone. In SCC patients, 8 had retinopathy alone and 8 had maculopathy alone. No association between the presence of retinopathy and maculopathy was detected.

AI was higher and RBC deformability lower at $3 \mathrm{~Pa}$ in SCA compared to SCC patients. Blood viscosity was higher in SCC patients than in SCA. MCV and LDH were higher and hemoglobin, RBC count and hematocrit lower in SCA than in SCC patients. WBC, platelet count and CRP were 
Table 1

Comparison of general characteristics, hematological and hemorheological parameters between SCA and SCC patients

\begin{tabular}{|c|c|c|}
\hline & $\operatorname{SCA}(n=37)$ & $\operatorname{SCC}(n=33)$ \\
\hline Retinopathy (\%) & 51.3 & 34.4 \\
\hline Maculopathy (\%) & 47.1 & 34.5 \\
\hline Age (years) & $40.3 \pm 13.4$ & $40.3 \pm 2.3$ \\
\hline Gender (M/F) & $17 / 20$ & $11 / 22$ \\
\hline $\mathrm{HbF}(\%)$ & $9.3 \pm 1.1$ & $1.5 \pm 0.2^{* *}$ \\
\hline Hydroxyurea (\%) & 59.4 & - \\
\hline$\alpha$-thalassemia $(\%)$ & 45.9 & 27.3 \\
\hline WBC $\left(10^{9} / \mathrm{L}\right)$ & $8.05 \pm 0.4$ & $7.51 \pm 0.51$ \\
\hline $\operatorname{RCBs}\left(10^{12} / \mathrm{L}\right)$ & $2.7 \pm 0.1$ & $4.3 \pm 0.1 * *$ \\
\hline $\mathrm{Hb}(\mathrm{g} / 100 \mathrm{ml})$ & $8.3 \pm 0,2$ & $10.7 \pm 0.2 * *$ \\
\hline $\operatorname{MCV}(f l)$ & $90.6 \pm 1.9$ & $72.9 \pm 1.4 * *$ \\
\hline Platelets count $\left(10^{9} / \mathrm{L}\right)$ & $320 \pm 17.5$ & $330.4 \pm 30.8$ \\
\hline Lactate dehydrogenase (UI/l) & $508 \pm 22$ & $265 \pm 13^{* *}$ \\
\hline CRP (mg/L) & $12.1 \pm 2.9$ & $9.9 \pm 2.4$ \\
\hline $\operatorname{Hct}(\%)$ & $24.5 \pm 0.7$ & $30.8 \pm 0.6^{* *}$ \\
\hline Blood viscosity (cP) at $90 \mathrm{~s}^{-1}$ & $5.6 \pm 0.3$ & $7.3 \pm 0.5^{*}$ \\
\hline Blood viscosity (cP) at $225 \mathrm{~s}^{-1}$ & $4.9 \pm 0.3$ & $5.6 \pm 0.3^{*}$ \\
\hline $\mathrm{AI}(\%)$ & $59 \pm 3$ & $48 \pm 3 *$ \\
\hline$\gamma\left(s^{-1}\right)$ & $382 \pm 44$ & $315 \pm 39$ \\
\hline $\mathrm{RBC}$ deformability (a.u.) at $3 \mathrm{~Pa}$ & $0.18 \pm 0.01$ & $0.23 \pm 0.01 *$ \\
\hline $\mathrm{RBC}$ deformability (a.u) at $30 \mathrm{~Pa}$ & $0.44 \pm 0.08$ & $0.41 \pm 0.01$ \\
\hline
\end{tabular}

WBC: white blood cell count; RBC: red blood cell count; PLT: platelet count; Hb: hemoglobin concentration; Hct: hematocrit; MCV: mean cell volume; hemoglobin; CRP: C-reactive protein; LDH: lactate dehydrogenase; AI: red blood cell aggregation index; $\gamma$ : red blood cell disaggregation threshold. Statistical difference between the two groups: $* p<0.05 ; * * p<0.01$.

not statistically different between the two SCD patient groups. SCA patients showed a higher rate of hemoglobin F than SCC patients. Age, alpha-thalassemia frequency and gender did not differ between SCA and SCC patients (Table 1). Frequencies of each medical complication in SCA and SCC groups are displayed in Table 2. We observed that only the frequency of leg ulcers was significantly higher in SCA than in SCC patients.

No link was found between maculopathy and retinopathy and the complications studied. Alphathalassemia frequency was not different between those with ocular complications and those without (Tables 3 and 4). We found no association between hydroxyurea treatment and maculopathy or retinopathy (Table 3 ).

We then compared the different hematological and hemorheological parameters between SCA or SCC patients with or without maculopathy/retinopathy. The values are reported in the Tables 3 to 6 and no difference was found in any of the biological parameters investigated.

\section{Discussion}

Retinopathy is usually reported to be more frequent in SCC than in SCA patients [3, 19]. The high blood viscosity usually reported in SCC patients in comparison with SCA patients has been 
Table 2

Comparison of the clinical complications frequencies between SCA and SCC patients (\%)

\begin{tabular}{lcc}
\hline & SCA $(n=37)$ & $\operatorname{SCC}(n=33)$ \\
\hline Maculopathy (\%) & 47 & 34 \\
Retinopathy (\%) & 51 & 34 \\
Leg ulcers (\%) & 21 & $3 *$ \\
Osteonecrosis (\%) & 32 & 23 \\
Nephropathy (\%) & 49 & 27 \\
Acute chest syndrome (\%) & 13 & 3 \\
Vaso-occlusive crisis (\%) & 43 & 27 \\
\hline
\end{tabular}

Statistical difference between the two groups: $* p<0.05$.

Table 3

Comparison of general characteristics, hematological and hemorheological parameters between SCA patients with maculopathy and SCA patients without maculopathy

\begin{tabular}{lcc}
\hline & SCA with maculopathy & SCA without maculopathy \\
\hline Age & $41.57 \pm 2.04$ & $40.33 \pm 2.04$ \\
Gender (M/F) & $6 / 9$ & $10 / 8$ \\
HbF $(\%)$ & $9.50 \pm 1.14$ & $9.48 \pm 1.12$ \\
Hydroxyurea (\%) & 68.75 & 61.11 \\
$\alpha$-thalassemia (\%) & 37 & 44 \\
WBC $\left(10^{9} / \mathrm{L}\right)$ & $7.9 \pm 0.5$ & $8.02 \pm 0.49$ \\
RCBs $\left(10^{12} / \mathrm{L}\right)$ & $2.8 \pm 0.2$ & $2.7 \pm 0.1$ \\
$\mathrm{Hb}(\mathrm{g} / 100 \mathrm{ml})$ & $8.6 \pm 0.3$ & $8.2 \pm 0.3$ \\
MCV (fl) & $91.28 \pm 1.95$ & $91.05 \pm 1.90$ \\
Platelets count $\left(10^{9} / \mathrm{L}\right)$ & $317.1 \pm 18.4$ & $324.1 \pm 17.6$ \\
Lactate dehydrogenase (UI/l) & $498 \pm 22$ & $502 \pm 22$ \\
Hct $(\%)$ & $24.94 \pm 1.02$ & $24.16 \pm 0.97$ \\
Blood viscosity (cP) at $90 \mathrm{~s}^{-1}$ & $5.6 \pm 0.4$ & $5.8 \pm 0.3$ \\
Blood viscosity (cP) at $225 \mathrm{~s}^{-1}$ & $4.9 \pm 0.2$ & $4.9 \pm 0.2$ \\
AI $(\%)$ & $57 \pm 3$ & $57 \pm 3$ \\
$\gamma\left(\mathrm{s}^{-1}\right)$ & $366 \pm 43$ & $359 \pm 45$ \\
RBC deformability (a.u.) at 3 Pa & $0.22 \pm 0.01$ & $0.23 \pm 0.01$ \\
RBC deformability (a.u) at 30 Pa & $0.44 \pm 0.02$ & $0.45 \pm 0.02$ \\
\hline
\end{tabular}

WBC: white blood cell count; RBC: red blood cell count; PLT: platelet count; Hb: hemoglobin concentration; Hct: hematocrit; MCV: mean cell volume; hemoglobin; LDH: lactate dehydrogenase; AI: red blood cell aggregation index; $\gamma$ : red blood cell disaggregation threshold.

hypothesized to be involved in the increased risk of SCC patients to develop retinopathy [20]. In our study, SCC patients had higher blood viscosity than SCA. Despite this difference, the frequency of retinopathy was identical between SCC and SCA patients. Moreover, it has been demonstrated that high hemoglobin concentration and low fetal hemoglobin level could increase the risks of SCA patients to develop sickle retinopathy $[7,10]$. In our study, SCA patients showed a higher rate of fetal hemoglobin than SCC patients but we found no association between hematological or hemorheological parameters and maculopathy or retinopathy. Increased blood viscosity has been suspected to increase the risk for severe proliferative sickle retinopathy (PSR), notably in SCC patients [5]. However, we found no 
Table 4

Comparison of general characteristics, hematological and hemorheological parameters between SCC patients with maculopathy and SCC patients without maculopathy

\begin{tabular}{|c|c|c|}
\hline & SCC with maculopathy & SCC without maculopathy \\
\hline Age & $41.7 \pm 2.4$ & $40.1 \pm 2.5$ \\
\hline Gender (M/F) & $4 / 6$ & $7 / 13$ \\
\hline $\mathrm{HbF}(\%)$ & $1.63 \pm 0.26$ & $1.55 \pm 0.25$ \\
\hline$\alpha$-thalassemia $(\%)$ & 37 & 46 \\
\hline $\mathrm{WBC}\left(10^{9} / \mathrm{L}\right)$ & $7.4 \pm 0.5$ & $7.3 \pm 0.5$ \\
\hline $\operatorname{RCBs}\left(10^{12} / \mathrm{L}\right)$ & $4.3 \pm 0.2$ & $4.2 \pm 0.1$ \\
\hline $\mathrm{Hb}(\mathrm{g} / 100 \mathrm{ml})$ & $10.6 \pm 0.4$ & $10.7 \pm 0.3$ \\
\hline $\operatorname{MCV}(f l)$ & $73.1 \pm 2.5$ & $72.8 \pm 1.7$ \\
\hline Platelets count $\left(10^{9} / \mathrm{L}\right)$ & $329.3 \pm 29.2$ & $323.1 \pm 31.9$ \\
\hline Lactate dehydrogenase (UI/l) & $264 \pm 12$ & $264 \pm 14$ \\
\hline $\operatorname{Hct}(\%)$ & $30.7 \pm 1.1$ & $30.8 \pm 0.7$ \\
\hline Blood viscosity $(\mathrm{cP})$ at $90 \mathrm{~s}^{-1}$ & $6.8 \pm 0.3$ & $7.4 \pm 0.5$ \\
\hline Blood viscosity $(\mathrm{cP})$ at $225 \mathrm{~s}^{-1}$ & $5.4 \pm 0.3$ & $5.5 \pm 0.3$ \\
\hline $\mathrm{AI}(\%)$ & $49 \pm 3$ & $48 \pm 3$ \\
\hline$\gamma\left(\mathrm{s}^{-1}\right)$ & $330 \pm 44$ & $316 \pm 41$ \\
\hline $\mathrm{RBC}$ deformability (a.u.) at $3 \mathrm{~Pa}$ & $0.18 \pm 0.01$ & $0.18 \pm 0,01$ \\
\hline $\mathrm{RBC}$ deformability (a.u) at $30 \mathrm{~Pa}$ & $0.41 \pm 0,02$ & $0.42 \pm 0.02$ \\
\hline
\end{tabular}

WBC: white blood cell count; RBC: red blood cell count; PLT: platelet count; Hb: hemoglobin concentration; Hct: hematocrit; MCV: mean cell volume; hemoglobin; LDH: lactate dehydrogenase; AI: red blood cell aggregation index; $\gamma$ : red blood cell disaggregation threshold.

Table 5

Comparison of general characteristics, hematological and hemorheological parameters between SCA patients with retinopathy and SCA patients without retinopathy

\begin{tabular}{lcc}
\hline & SCA with retinopathy & SCA without retinopathy \\
\hline Age & $41.12 \pm 2.11$ & $40.33 \pm 2.04$ \\
Gender (M/F) & $8 / 11$ & $9 / 9$ \\
HbF $(\%)$ & $9.5 \pm 1.1$ & $9.5 \pm 1.1$ \\
Hydroxyurea (\%) & 68.4 & 47.1 \\
$\alpha$-thalassemia (\%) & 41 & 60 \\
WBC $\left(10^{9} / \mathrm{L}\right)$ & $7.92 \pm 0.49$ & $8.02 \pm 0.49$ \\
RCBs $\left(10^{12} / \mathrm{L}\right)$ & $2.8 \pm 0.2$ & $2.7 \pm 0.1$ \\
Hb $(\mathrm{g} / 100 \mathrm{ml})$ & $8.3 \pm 0.3$ & $8.4 \pm 0.3$ \\
MCV (fl) & $91.28 \pm 1.95$ & $91.05 \pm 1.90$ \\
Platelets count $\left(10^{9} / \mathrm{L}\right)$ & $317.1 \pm 18.4$ & $324.1 \pm 17.6$ \\
Lactate dehydrogenase $(\mathrm{UI} / \mathrm{l})$ & $498 \pm 21$ & $502 \pm 22$ \\
Hct $(\%)$ & $24.5 \pm 1.1$ & $24.5 \pm 0.9$ \\
Blood viscosity (cP) at $90 \mathrm{~s}^{-1}$ & $5.7 \pm 0.3$ & $5.6 \pm 0.4$ \\
Blood viscosity (cP) at $225 \mathrm{~s}^{-1}$ & $4.9 \pm 0.2$ & $4.8 \pm 0.3$ \\
AI $(\%)$ & $57 \pm 2.5$ & $58 \pm 3$ \\
$\gamma\left(\mathrm{s}^{-1}\right)$ & $366 \pm 43$ & $383 \pm 46$ \\
RBC deformability (a.u.) at $3 \mathrm{~Pa}$ & $0.22 \pm 0.01$ & $0.23 \pm 0.01$ \\
RBC deformability (a.u) at $30 \mathrm{~Pa}$ & $0.44 \pm 0.02$ & $0.44 \pm 0.02$ \\
\hline
\end{tabular}

WBC: white blood cell count; RBC: red blood cell count; PLT: platelet count; Hb: hemoglobin concentration; Hct: hematocrit; MCV: mean cell volume; hemoglobin; LDH: lactate dehydrogenase; AI: red blood cell aggregation index; $\gamma$ : red blood cell disaggregation threshold. 
Table 6

Comparison of general characteristics, hematological and hemorheological parameters between SCC patients with retinopathy and SCC patients without retinopathy

\begin{tabular}{lcc}
\hline & SCC with retinopathy & SCC without retinopathy \\
\hline Age & $41.4 \pm 2.3$ & $40.3 \pm 2.3$ \\
Gender (M/F) & $4 / 7$ & $7 / 14$ \\
HBF (\%) & $1.6 \pm 0.3$ & $1.5 \pm 0.2$ \\
Hydroxyurea (\%) & 0 & 0 \\
$\alpha$-thalassemia (\%) & 27 & 28 \\
WBC $\left(10^{9} / \mathrm{L}\right)$ & $7.4 \pm 0.5$ & $7.5 \pm 0.5$ \\
RCBs $\left(10^{12} / \mathrm{L}\right)$ & $4.5 \pm 0.2$ & $4.1 \pm 0.1$ \\
Hb (g/100 ml) & $11.1 \pm 0.3$ & $10.5 \pm 0.3$ \\
MCV (fl) & $72.8 \pm 1.4$ & $72.3 \pm 1.4$ \\
Platelets count (10 $/ \mathrm{L})$ & $311.7 \pm 29.2$ & $330.4 \pm 30.8$ \\
Lactate dehydrogenase (UI/l) & $256 \pm 19$ & $265 \pm 13$ \\
Hct (\%) & $31.9 \pm 0.8$ & $30.2 \pm 0.8$ \\
Blood viscosity (cP) at $90 \mathrm{~s}^{-1}$ & $6.9 \pm 0.3$ & $7.3 \pm 0.5$ \\
Blood viscosity (cP) at $225 \mathrm{~s}^{-1}$ & $5.4 \pm 0.3$ & $5.7 \pm 0.5$ \\
AI (\%) & $47 \pm 3$ & $48 \pm 3$ \\
$\gamma\left(\mathrm{s}^{-1}\right)$ & $298 \pm 40$ & $316 \pm 41$ \\
RBC deformability (a.u.) at 3 Pa & $0.18 \pm 0.01$ & $0.18 \pm 0.01$ \\
RBC deformability (a.u) at 30 Pa & $0.41 \pm 0.02$ & $0.41 \pm 0.01$ \\
\hline
\end{tabular}

WBC: white blood cell count; RBC: red blood cell count; PLT: platelet count; Hb: hemoglobin concentration; Hct: hematocrit; MCV: mean cell volume; hemoglobin; LDH: lactate dehydrogenase; AI: red blood cell aggregation index; $\gamma$ : red blood cell disaggregation threshold.

difference in blood viscosity between patients with retinopathy and those without. Further studies in larger cohort are needed to understand the discrepancies between these different studies.

Our study found no association between sickle cell maculopathy and sickle cell genotype. While our SCA and SCC patients are very different regarding hematological and hemorheological parameters, the lack of genotype effect suggests that these biological factors could not play a key role in the development of maculopathy. Nevertheless, the pathophysiological mechanisms involved in retinopathy and maculopathy could be different and remain uncompletely understood. It has been suspected that prolonged episodes of ischemia could play a role in the development of ocular complications [3]. Using ocular coherence tomography angiography (OCT-A), several authors reported reduced flow within the superficial and deep macular plexuses [21, 22]. In our study, only 11 patients had both retinopathy and maculopathy. Spontaneous regression by auto-infarction of neovascular complexes is well described in sickle cell retinopathy and occurs most of the time two years after development of proliferative lesions [8]. Indeed, the absence of retinopathy does not mean that the patient did not experience this complication few years ago. In contrast, maculopathy appears in OCT as a persistent macular thinning [21]. Maculopathy and retinopathy may develop simultaneously both resulting from an ischemic origin. Nevertheless, the lack of age difference between patients with maculopathy and those with retinopathy does not suggest a chronological sequence in the appearance of retinopathy and maculopathy.

SCD maculopathy has sometimes been reported to be the consequence of central retina artery occlusion (CRAO) [23]. However, none of our patient had a history of CRAO, although we found $37,1 \%$ of asymptomatic maculopathy. The thinning may reflect the chronic ischemia of the retinal ganglion cells and nerve fibers coursing temporally as they head toward the optic nerve [13]. Impaired 
blood rheology in SCD plays an important role in the development of several complications, notably because of the resulting decrease in blood flow and tissue oxygenation [15]. Recently, Hussnain et al. suggested a paracentral acute maculopathy as a precursor of macular thinning in SCD [24]. We found no association between hemorheological or hematological parameters and SCD maculopathy. Indeed, at that time, it is difficult to decipher the exact mechanisms at the origin of maculopathy in SCD. Although never studied, we could suspect a role of inflammation, oxidative stress and endothelial dysfunction in the development of this complication, as it is the case in diabetes [25].

Our study showed that retinopathy and maculopathy are common in SCD. Nevertheless, we found no association with hematological parameters, blood rheology or genotype.

\section{References}

[1] Kim SY, Mocanu C, Mcleod DS, Bhutto IA, Merges C, Eid M, et al. Expression of pigment epithelium-derived factor (PEDF) and vascular endothelial growth factor (VEGF) in sickle cell retina and choroid. Exp Eye Res. 2003;77(4):43345.

[2] National Heart and Blood Institute Division of Blood Diseases and Resources. The management of sickle cell disease (fourth Edition, June 2002. Manag Sick cell Dis (Fourth Ed June 2002). 2002;95-7.

[3] Elagouz M, Jyothi S, Gupta B, Sivaprasad S. Sickle cell disease and the eye: Old and new concepts. Surv Ophthalmol. 2010;55(4):359-77. 10.1016/j.survophthal.2009.11.004

[4] Asdourian GK, Nagpal KC, Busse B, Goldbaum M, Patriankos D, Rabb MF, et al. Macular and perimacular vascular remodelling sickling haemoglobinopathies. Br J Ophthalmol. 1976;60(6):431-53.

[5] Lemaire C, Lamarre Y, Lemonne N, Waltz X, Chahed S, Cabot F, et al. Severe proliferative retinopathy is associated with blood hyperviscosity in sickle cell hemoglobin-C disease but not in sickle cell anemia. Clin Hemorheol Microcirc. 2013;55(2):205-12.

[6] Serjeant BE, Mason KP, Condon PI, Hayes RJ, Kenny MW, Stuart J, et al. Blood rheology and proliferative retinopathy in sickle cell-haemoglobin C disease. Br J Ophthalmol. 1984;68(5):325-8.

[7] Fox PD, Dunn DT, Morris JS, Serjeant GR. Risk factors for proliferative sickle retinopathy. Br J Ophthalmol. 1990;74(3):172-6.

[8] Goldberg MF. Classification and pathogenesis of proliferative sickle retinopathy. Am J Ophthalmol. 1971;71(3):649-65.

[9] Hayes RJ, Condon PI, Serjeant GR. Haematological factors associated with proliferative retinopathy in sickle cellhaemoglobin C disease. Br J Ophthalmol. 1981;65(10):712-7.

[10] Hayes RJ, Condon PI, Serjeant GR. Haematological factors associated with proliferative retinopathy in homozygous sickle cell disease. Br J Ophthalmol. 1981;65(1):29-35.

[11] Han IC, Tadarati M, Scott AW. Macular Vascular Abnormalities Identified by Optical Coherence Tomographic Angiography in Patients With Sickle Cell Disease. JAMA Ophthalmol. 2015;133(11):1337-40.

[12] Mathew R, Bafiq R, Ramu J, Pearce E, Richardson M, Drasar E, et al. Spectral domain optical coherence tomography in patients with sickle cell disease. Br J Ophthalmol. 2015;99(7):967-72.

[13] Murthy RK, Grover S, Chalam KV. Temporal Macular Thinning on Spectral-Domain Optical Coherence Tomography in Proliferative Sickle Cell Retinopathy. Arch Ophthalmol. 2011;129(2):247.

[14] Han IC, Tadarati M, Pacheco KD, Scott AW. Evaluation of Macular Vascular Abnormalities Identified by Optical Coherence Tomography Angiography in Sickle Cell Disease. Am J Ophthalmol. 2017;177:90-9.

[15] Connes P, Alexy T, Detterich J, Romana M, Hardy-Dessources M-D, Ballas SK. The role of blood rheology in sickle cell disease. Blood Rev. 2016;30(2):111-8.

[16] Baskurt OK, Boynard M, Cokelet GC, Connes P, Cooke BM, Forconi S, et al. New guidelines for hemorheological laboratory techniques. Clin Hemorheol Microcirc. 2009;42(2):75-97.

[17] Tan AS, Quah TC, Low PS, Chong SS. A rapid and reliable 7-deletion multiplex polymerase chain reaction assay for alpha-thalassemia. Blood. 2001;98(1):250-1.

[18] Liu J, Jia X, Tang N, Zhang X, Wu X, Cai R, et al. Novel technique for rapid detection of $\alpha$-globin gene mutations and deletions. Transl Res. 2010;155(3):148-55.

[19] Goldberg MF. Retinal vaso-occlusion in sickling hemoglobinopathies. Birth Defects Orig Artic Ser. 1976;12(3): 475-515.

[20] Chen S-N, Hwang J-F, Chen Y-T. Macular thickness measurements in central retinal artery occlusion by optical coherence tomography. Retina. 2011;31(4):730-7. 
[21] Sanfilippo CJ, Klufas MA, Sarraf D, Tsui I. Optical coherence tomography angiography of sickle cell maculopathy. Retin Cases Brief Rep. 2015;9(4):360-2.

[22] Jung JJ, Chen MH, Frambach CR, Rofagha S, Lee SS. Spectral domain versus swept source optical coherence tomography angiography of the retinal capillary plexuses in sickle cell maculopathy. Retin Cases Brief Rep. 2016;1.

[23] Fine, Petrovic, Irvine, Bhisitkul. Correction-spontaneous central retinal artery occlusion in hemoglobin SC disease(1). Am J Ophthalmol. 2000;130(6):906-7.

[24] Hussnain SA, Coady PA, Stoessel KM. Paracentral acute middle maculopathy: Precursor to macular thinning in sickle cell retinopathy. BMJ Case Rep. 2017;2017:bcr-2016-216124.

[25] Madsen-Bouterse SA, Kowluru RA. Oxidative stress and diabetic retinopathy: Pathophysiological mechanisms and treatment perspectives. Rev Endocr Metab Disord. 2008;9(4):315-27. 